\title{
Interactive comment on "Using spatial Markovian chain for the statistical analysis of seismic occurrences in the Azores Region” by M. C. M. Rodrigues and C. S. Oliveira
}

\author{
M. C. M. Rodrigues and C. S. Oliveira \\ mcmr@fct.unl.pt
}

Received and published: 4 July 2016

Comments to the referees will be given below point by point and whenever the authors felt that some parts or slight corrections should be added to the text, they are placed in red color. Referee \#11. Generally, more emphasize should be given in the introduction on the significance and implications of this work. R. We agree with this comment and added several sentences to the text for clarification.. 2. I find the statistical relation between the zones of background seismicity and the zones of high seismic rates, poor, with no dependency; the only reason that the probability of the next event in Zone 5 is high for these background zones, is because Zone 5 is the most active in any case. This is different from the conclusion and discussion of the authors. As I see

Printer-friendly version

Discussion paper 
it, the tool is good, but its analysis should be improved. R. The phase: "Furthermore, the region 5 has the largest number of occurrences" had been added. In fact, the first-step transition is concentrated essentially in zone 5 for most cases. However, for second and more-step transitions the situation may change the situation of from zone 5 to a background zone with low probability. This text was added to the paper. 3. The tool does not take into account magnitudes! It seems that it works well without including it, but I reckon that by adding magnitude you could get better understanding of the seismic zones. Authors should either add the magnitude to their analysis, or consider the magnitudes in their discussion: What do they expect? How would the addition of magnitudes affect their results? R. As is said in "Methodology", this study is a component of an ongoing study for modelling the seismic process of occurrences in the Azores region. The first step was the definition of seismic zones, after we are exploring the spatial sequence of epicenters among the zones already defined. The introduction of magnitude and time may be not treated only with Markovian chains, but with conditioned distribution functions with more than one variable, for example, magnitude conditioned by time and space, and this is too long to be included in a single paper. We agree with this reviewer that the variable magnitude is very important in the whole process. Q. N-step transitions are also analyzed, and Markovian chains are applied for this purpose". Please clarify and expand! R. We also want to quantify the influence of the location of an earthquake not only on the next earthquake but after $\mathrm{N}$ seismic events. $\mathrm{N}$-step transitions are also analyzed, and Markovian chains are applied for this purpose. This is "how seismicity moves from zone to zone". Q. "Traditionally, seismic phenomena have been described using Poisson models". To the best of my knowledge, there are many ways of analyzing the seismic phenomena, by either statistical or physical manner. Poisson models are one of many descriptions. $\mathrm{R}$. Poisson models are based on the assumption that an event can occur at random and the number of occurrences in non-overlapping intervals is independent. This is the "lack of memory" of Poisson processes. An occurrence at an instant in time is not affected by past occurrences nor does affect any future events. In reality the

Interactive comment
Printer-friendly version

Discussion paper 
occurrence process has memory and consequently "memory" models are better to describe the process. Other smaller issues: Chi-square two-sample test is a goodness of fit test, is mentioned in statistic bibliography and it is used to check is two samples may be picked from the same population. We include another figure with zoom for a better visualization of the epicenter locations Same explanations or corrections have been made in text in red color. Referee \#2 R. The comments provided by referee \#2 are based in the fact that the paper is very much mathematical oriented, without the physical background. The authors do not agree with this argument because all the paper is aiming at reproduce the reality of the occurrences of earthquakes independently of any underlying model. We select Markovian chains because they seem to better represent that reality. The Title itself tries to express this view. Of course the results obtained show that the physical models are present in all that as described in the Space-variable analysis. (section 4.1) The interpretation of $T$ matrix (eq. 3 ) is made in responding to referee \#1 Interpretations of the mathematical models used were added to the manuscript (in red color). Please note that the results are consistent with the seismic characteristics of the Azores region and its geological structures.

Please also note the supplement to this comment: http://www.nat-hazards-earth-syst-sci-discuss.net/nhess-2016-113/nhess-2016-113AC2-supplement.pdf

Interactive comment on Nat. Hazards Earth Syst. Sci. Discuss., doi:10.5194/nhess-2016-113, 2016. 